\title{
Utilisation optimale de l'assistance respiratoire extracorporelle durant la pandémie de COVID-19 : considérations pratiques en contexte canadien
}

\author{
Ken Kuljit S. Parhar MD MSc, Laurance Lequier MD, Jaime Blackwood MD, Danny J. Zuege MD MSc, \\ Gurmeet Singh MD MSc
}

E Citation : CMAJ 2020 April 6;192:E372-4. doi : 10.1503/cmaj.200448-f; diffusion hâtive le 26 mars 2020

Voir la version anglaise de l'article ici : www.cmaj.ca/lookup/doi/10.1503/cmaj.200448

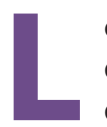

es patients infectés par le nouveau coronavirus du syndrome respiratoire aigu sévère 2 (SRAS-CoV-2) peuvent demeurer asymptomatiques ou développer la maladie à coronavirus 2019 (COVID-19), caractérisée par une infection et une inflammation des voies respiratoires inférieures ${ }^{1}$. Environ $14 \%$ des personnes atteintes présentent des symptômes graves, comme un syndrome de détresse respiratoire aiguë². Dans ses lignes directrices ${ }^{3}$, l'Organisation mondiale de la Santé recommande d'envisager l'assistance respiratoire extracorporelle (AREC), une technique d'assistance cardiopulmonaire invasive qui consiste à créer un circuit d'échange d'oxygène et de dioxyde de carbone à l'extérieur du corps ${ }^{4}$, pour les patients atteints de la COVID-19 dont l'hypoxémie est réfractaire à la ventilation pulmonaire protectrice. L'AREC est un traitement de sauvetage destiné aux patients dont on n'a pu stabiliser les fonctions vitales autrement et qui en ont besoin pour survivre. Elle nécessite beaucoup de ressources, qui souvent sont limitées même en l'absence de pandémie. Dans cet article, nous traiterons de l'utilité de l'AREC dans les cas de pneumonie virale et décrirons comment l'utilisation des ressources associées est optimisée en Alberta (Canada) à l'ère de la pandémie, dans l'espoir que nos façons de faire puissent servir dans d'autres provinces et territoires.

Le recours à l'AREC a connu une croissance substantielle dans la dernière décennie, principalement pour 2 raisons : 1 ) l'essai clinique randomisé CESAR (Conventional Ventilatory Support Versus Extracorporeal Membrane Oxygenation for Severe Adult Respiratory Failure [comparaison de la ventilation classique et de l'oxygénation extracorporelle chez les adultes ayant une insuffisance respiratoire grave]) a démontré que l'orientation des patients atteints d'insuffisance respiratoire vers un centre où l'on pratique l'AREC augmentait leur taux de survie'; et 2) la pandémie de grippe due à l'émergence de la nouvelle souche grippale $A(H 1 N 1)$. L'AREC a fait ses preuves dans plusieurs pays lors de la pandémie de grippe H1N1. L'Australian and New Zealand Intensive Care Society [la Société australienne et néo-

\section{POINTS CLÉS}

- La préparation à la pandémie de COVID-19 demande une approche multidisciplinaire et dirigée par ceux qui y participent, étant donné qu'environ $14 \%$ des personnes atteintes présentent des symptômes graves, comme un syndrome de détresse respiratoire aiguë.

- Dans ses lignes directrices, l'Organisation mondiale de la Santé recommande d'envisager l'assistance respiratoire extracorporelle (AREC), une ressource rare qui pourrait se voir rationnée en contexte de pandémie, pour les patients atteints de la COVID-19 dont l'hypoxémie est réfractaire à la ventilation pulmonaire protectrice.

- Il est essentiel d'avoir une stratégie de communication limpide et exacte de la capacité en matière d'AREC; en Alberta, nous avons conçu un modèle représentant la capacité et le niveau de préparation par les couleurs verte, jaune et rouge.

- Dans les régions étendues, qu'on trouve dans bon nombre des provinces et territoires canadiens, il est important de bien définir le processus de recommandation pour chaque établissement d'origine, et les services d'AREC pour adultes et pour enfants pourraient avoir à s'entraider.

- Si la demande de ressources aux soins intensifs devient excessive, il faudra suspendre les services d'AREC et réaffecter l'ensemble des ressources de soins intensifs disponibles en fonction des besoins.

zélandaise de soins intensifs] a calculé un taux de survie supérieur à $70 \%$ chez les patients dont la grippe H1N1 a évolué en syndrome de détresse respiratoire aiguë et qui ont reçu une $A R E C^{6}$. Une étude réalisée au Royaume-Uni a fait état d'une réduction de la mortalité chez les patients présentant un syndrome de détresse respiratoire aiguë dû à la grippe H1N1 qui ont reçu une AREC par rapport à des témoins jumelés selon le score de propension 7 . Un groupe de recherche français n'a observé aucune amélioration sur le plan de la mortalité ${ }^{8}$, mais cette discordance s'explique probablement par des différences méthodologiques et l'hétérogénéité des patients non jumelés. 
La pandémie de COVID-19 que nous connaissons actuellement est la troisième épidémie de maladie à coronavirus à prendre une ampleur mondiale dans l'histoire récente, après celle du syndrome respiratoire aigu sévère (SRAS) et du syndrome respiratoire du Moyen-Orient (SRMO). L'AREC était encore peu utilisée au moment de la pandémie de SRAS. Durant l'épidémie de SRMO, des petites séries de cas ont révélé une corrélation positive entre le recours à l'AREC et la probabilité de survie ${ }^{9}$, à partir de laquelle on peut raisonnablement présumer que l'intervention aiderait certains patients atteints de la COVID-19 qui ont développé un syndrome de détresse respiratoire aiguë sévère à s'en sortir. Jusqu'à maintenant, la progression rapide de la pandémie de COVID-19 n'a laissé qu'une quantité minime de données d'observation et peu de temps pour mener des études d'efficacité rigoureuses. De vastes études observationnelles multicentriques, comme l'étude ECMOCARD (Extracorporeal Membrane Oxygenation for 2019 Novel Coronavirus Acute Respiratory Disease [oxygénation extracorporelle dans les cas de maladie respiratoire aiguë à coronavirus 2019]), sont en cours, et la collecte de données en temps réel nous en dira plus sur l'utilité de l'AREC dans les cas de COVID-19.

Les pandémies créent généralement un afflux de patients aux soins intensifs, dont les besoins peuvent rapidement excéder la capacité du système ${ }^{10}$. Si, d'un côté, les efforts de la santé publique visant à ralentir la transmission de personne à personne peuvent limiter la pression sur les soins intensifs ${ }^{11}$, de l'autre, chaque infection ou quarantaine d'un fournisseur de soins de santé a l'effet inverse, et vient réduire les ressources en soins intensifs, y compris la capacité à fournir une AREC. Idéalement, il faudrait centraliser le triage et l'affectation des ressources pour assurer leur répartition juste et équitable dans un contexte où les ressources destinées aux soins intensifs sont en forte demande et les possibilités d'approvisionnement, limitées.

Il est essentiel d'avoir une stratégie de communication limpide et exacte de la capacité en matière d'AREC. Cette capacité dépend du nombre de patients qui bénéficieraient de l'intervention à un moment donné ainsi que du nombre de patients qui peuvent se faire poser des canules à cet effet compte tenu de la disponibilité des articles à usage unique. En Alberta, nous avons conçu un modèle simple représentant 3 niveaux de préparation par les couleurs verte, jaune et rouge (figure supplémentaire 1 de l'annexe 1 [en anglais], au www.cmaj.ca/lookup/ suppl/doi:10.1503/cmaj.200448/-/DC1). Le niveau vert indique que la capacité à fournir une AREC et la quantité de matériel à usage unique disponible suffisent pour poursuivre les activités sans nuire aux services concernés, par exemple les soins intensifs et la chirurgie cardiaque. Le niveau jaune représente l'intervalle de capacité et de stocks pouvant commander un rationnement. Quant au niveau rouge, il correspond à l'atteinte de la capacité maximale ou d'un seuil de stock critique, et a des répercussions importantes sur les activités aux soins intensifs et en chirurgie cardiaque. Mais, quel que soit le niveau, des procédures adaptées prévoyant différents parcours de soins selon le nombre de spécialistes de l'AREC et de perfusionnistes sur le terrain doivent être mises en place.
La collaboration entre les services albertains d'AREC pour adultes et pour enfants favoriserait le partage de l'équipement, des effectifs et du matériel à usage unique. De plus, acheter le matériel en gros permet d'assurer une bonne répartition entre les services et, tant que l'approvisionnement n'est pas interrompu, le maintien de stocks suffisants et la satisfaction de la demande clinique.

Par souci d'équité et d'efficacité, les indications de l'AREC devraient être définies et diffusées à l'avance. En Alberta, une partie du groupe de préparation à la pandémie de COVID-19, lequel est membre du Critical Care Strategic Clinical Network [Réseau clinique stratégique de soins intensifs] de la province (tableau supplémentaire 1 de l'annexe 1), a proposé une démarche de sélection des patients fondée sur des principes. Chaque cas devrait être évalué en premier lieu par un consultant en AREC, qui, en recommandant des options thérapeutiques à essayer avant d'envisager un transfert vers un centre où l'on pratique l'AREC ou la pose de canules, pourrait optimiser le traitement médical conventionnel. Nous croyons qu'avoir un consultant en AREC de garde 24 heures sur 24 et 7 jours sur 7 devrait être la norme. Les patients qui sont âgés, qui ont des comorbidités graves ou nombreuses et qui ne retrouveront probablement pas une fonction pulmonaire suffisante pour respirer par eux-mêmes ne devraient pas être considérés pour I'AREC, car celle-ci risque de ne pas leur apporter plus de bienfaits que la prise en charge conventionnelle. Les patients ventilés mécaniquement pendant plus de 7 jours sont peu susceptibles de retrouver leur fonction pulmonaire originale, même avec une AREC de sauvetage ${ }^{4}$. Le moment où transférer un patient pour qu'il reçoive une AREC doit être déterminé en fonction des risques liés au transport de malades dans un état critique, comme les conditions météorologiques, la durée du transport et la stabilité de l'état de santé du patient, puisqu'il est préférable que l'installation des canules se fasse à l'établissement qui pratique l'intervention. Sans compter qu'il est vraisemblablement impossible de libérer des équipes de transport pour qu'elles posent les canules avant l'arrivée à l'établissement d'accueil en contexte de pandémie et de grande sollicitation des ressources. La situation peut être évaluée au cas par cas, sous réserve de la disponibilité des ressources (tableau supplémentaire 2 de l'annexe 1 ).

Dans les régions de vaste superficie, qu'on trouve dans bon nombre des provinces et territoires canadiens, il est important de bien définir le processus de recommandation pour chaque établissement d'origine. Dans les circonstances exceptionnelles où un centre viendrait à en manquer, les services d'AREC pour adultes et pour enfants pourraient partager de l'équipement, des fournitures à usage unique et les services de spécialistes de I'AREC et de perfusionnistes.

L'évolution de la demande de ressources pourrait appeler une limitation graduelle de l'accessibilité de l'AREC, jusqu'à des restrictions strictes en cas de pénurie relative. En temps de pandémie, l'éthique veut généralement qu'on suive des principes utilitaristes, c'est-à-dire qu'on attribue, réaffecte ou réserve les ressources aux personnes à qui elles profiteront le plus $^{12}$. La priorité est souvent accordée aux patients ayant le 
meilleur pronostic, ce qui requiert un resserrement des critères d'acceptation et une adaptation des critères de rejet habituels. Enfin, il faut aussi tenir compte de la baisse de capacité attribuable à l'attrition, à la consommation de fournitures ou la surutilisation associée à la forte demande.

L'Alberta s'est dotée d'un système de triage et d'exploitation adéquate des services d'AREC, divisé en 4 paliers (tableau supplémentaire 3 de l'annexe 1) : le premier indique une viabilité des services à long terme; le deuxième, une pression accrue sur les ressources empêchant l'accès à l'intervention selon des critères élargis; le troisième, une pression élevée nécessitant l'accès exclusif à l'AREC aux patients ayant la meilleure probabilité de survie; et le quatrième, une demande excessive de ressources dans les soins intensifs qui déclenche la suspension des services d'AREC et la réaffectation de l'ensemble des ressources de soins intensifs disponibles aux autres patients qui se trouvent dans un état critique. La décision de passer d'un palier à un autre est prise par un centre de commande d'urgence centralisé qui examine en un tout l'information à jour concernant la demande et la capacité fournie par les services d'AREC et de soins intensifs.

En résumé, la préparation à la pandémie de COVID-19 demande une approche multidisciplinaire, dirigée par ceux qui y participent. L'AREC, une technique onéreuse et rare, mais qui sauve des vies, doit être utilisée de manière rationnelle pour que le plus grand nombre de patients qui en tireraient profit y aient accès.

\section{Références}

1. Zhu N, Zhang D, Wang W, et al. A novel coronavirus from patients with pneumonia in China, 2019. N Engl J Med 2020;382:727-33.

2. Wu Z, McGoogan JM. Characteristics of and important lessons from the coronavirus disease 2019 (COVID-19) outbreak in China: summary of a report of 72314 cases from the Chinese Center for Disease Control and Prevention. JAMA 24 février 2020. [Cyberpublication avant impression]. doi: 10.1001/jama.2020.2648.

3. Clinical management of severe acute respiratory infection when novel coronavirus ( $\mathrm{nCoV}$ ) infection is suspected [interim guidance]. Genève : Organisation mondiale de la Santé; 2020.

4. Parhar K, Vuylsteke A. What's new in ECMO: scoring the bad indications. Intensive Care Med 2014;40:1734-7.

5. Peek GJ, Mugford M, Tiruvoipati R, et al. Efficacy and economic assessment of conventional ventilatory support versus extracorporeal membrane oxygenation for severe adult respiratory failure (CESAR): a multicentre randomised controlled trial. Lancet 2009;374:1351-63.

6. Australia and New Zealand Extracorporeal Membrane Oxygenation Influenza (ANZ ECMO) Investigators; Davies A, Jones D, Bailey M, et al. Extracorporeal membrane oxygenation for 2009 influenza $A(H 1 N 1)$ acute respiratory distress syndrome. JAMA 2009;302:1888-95.

7. Noah MA, Peek GJ, Finney SJ, et al. Referral to an extracorporeal membrane oxygenation center and mortality among patients with severe 2009 influenza A(H1N1). JAMA 2011;306:1659-68.

8. Pham $\mathrm{T}$, Combes $\mathrm{A}$, Roze $\mathrm{H}$, et al. Extracorporeal membrane oxygenation for pandemic influenza $A(\mathrm{H} 1 \mathrm{~N} 1)$-induced acute respiratory distress syndrome: a cohort study and propensity-matched analysis. Am J Respir Crit Care Med 2013;187:276-85.

9. Alshahrani MS, Sindi A, Alshamsi F, et al. Extracorporeal membrane oxygenation for severe Middle East respiratory syndrome coronavirus. Ann Intensive Care 2018;8:3.

10. Grasselli G, Pesenti A, Cecconi M. Critical care utilization for the COVID-19 outbreak in Lombardy, Italy. JAMA 13 mars 2020 [Cyberpublication avant impression]. doi:10.1001/jama.2020.4031.

11. Murthy S, Gomersall CD, Fowler RA. Care for critically ill patients with COVID-19. JAMA 13 mars 2020. [Cyberpublication avant impression]. doi: 10.1001/jama.2020.3633.

12. Kaposy C, Bandrauk N, Pullman D, et al. Adapting the Hamilton Health Sciences critical care pandemic triage protocol. Healthc Q 2010;13:60-3.

\begin{abstract}
Intérêts concurrents : Gurmeet Singh déclare avoir été payé par Edwards Lifesciences pour ses services-conseils en tant que membre de son conseil consultatif. Ken Parhar est membre du comité consultatif d'Elsius Biomedical, mais n'a reçu aucune compensation directe ou indirecte. Le Dr Parhar a reçu des bourses de recherche des Instituts de recherche en santé du Canada et de la M.S.I. Foundation. Aucun autre intérêt concurrent déclaré.
\end{abstract}

Cet article a été commandé et n'a pas été révisé par les pairs.

Affiliations : Département de médecine de soins intensifs (Parhar, Zuege), Université de Calgary et Services de santé de l'Alberta, Calgary, Alta.; Département de pédiatrie, Division des soins intensifs (Lequier), Université de l'Alberta et Services de santé de l'Alberta, Edmonton, Alta.; Département de pédiatrie -
Division des soins intensifs (Blackwood), Université de Calgary et Services de santé de l'Alberta; Critical Care Strategic Clinical Network (Zuege), Services de santé de l'Alberta, Calgary, Alta.; Département de médecine de soins intensifs et de chirurgie, Division de la chirurgie cardiaque (Singh), Université de l'Alberta et Services de santé de l'Alberta, Edmonton, Alta.

Collaborateurs : Ken Kuljit Parhar et Gurmeet Singh ont contribué à la conception et à l'organisation du travail, et à la rédaction du premier jet. Tous les auteurs ont participé à la rédaction du manuscrit et en ont révisé de façon critique le contenu intellectuel important. Tous ont aussi approuvé la version finale pour publication et accepté d'assumer l'entière responsabilité de tous les aspects du travail.
Remerciements : Les auteurs remercient Nicole Dubyk, Don Granoski, Steve Menzies et Tanya Spence, coordonnateurs des services d'AREC, d'avoir lu le manuscrit et formulé leurs suggestions. Ils remercient également la présidente du comité, Kristin Robertson, et les membres du groupe de préparation à la pandémie de COVID-19 qui se consacre à l'AREC, des Services de santé de l'Alberta, pour leur apport aux documents de préparation à la pandémie. Pour terminer, ils veulent surtout souligner le savoirfaire et le dévouement des spécialistes de l'AREC, des perfusionnistes et de tous les autres professionnels de la santé qui font partie de la grande équipe nécessaire pour offrir aux malades qui sont dans un état critique les moyens techniques de leur sauver la vie.

Correspondance : Ken Kuljit Singh Parhar, ken.parhar@albertahealthservices.ca 